EXTENDED REPORT

\title{
Close association between valvar heart disease and central nervous system manifestations in the antiphospholipid syndrome
}

\author{
I Krause, S Lev, A Fraser, M Blank, M Lorber, L Stojanovich, J Rovensky, J Chapman, \\ Y Shoenfeld
}

See end of article for authors' affiliations

Correspondence to

Correspondence to:
Professor Y Shoenfeld Department of Medicine B Sheba Medical Centre, TelHashomer 52621, Israel; Shoenfel@post.tau.ac.il

Accepted 14 March 2005 Published Online First 18 March 2005

\begin{abstract}
Background: Heart valves lesions and central nervous system involvement are among the most common manifestations of the antiphospholipid syndrome (APS).

Objective: To evaluate possible interrelations between these manifestations in a large group of APS patients.

Methods: 284 APS patients were evaluated retrospectively, 159 of whom had primary APS. Cardiac-CNS associations were determined for the entire study population, and for subgroups of patients with primary APS or APS associated with systemic lupus erythematosus (SLE).

Results: Significant associations where found between cardiac vegetations and epilepsy $(p<0.02)$, and between cardiac valve thickening or dysfunction and migraine $(p=0.002)$. Borderline association was found between valvar vegetations and migraine $(p=0.09)$. A significant association was also found between all valvar lesions and stroke or transient ischaemic attacks. Subanalyses showed that patients with primary APS had significant associations between cardiac valve pathology and all CNS manifestations, while patients with APS associated with SLE had no such associations.

Conclusions: The study suggests potential differences in biological behaviour between primary APS and APS associated with SLE. The presence of cardiac valve pathology may be a risk factor for several types of CNS involvement in PAPS.
\end{abstract}

$\mathrm{T}$ raditionally described as a syndrome consisting of recurrent fetal loss, vascular thrombosis, and anticardiolipin autoantibodies, ${ }^{1-3}$ the antiphospholipid syndrome (APS) is now increasingly recognised as a multisystem disease, the clinical expression of which may include cardiac, neurological, renal, gastrointestinal, haematological, or cutaneous manifestations. ${ }^{45}$ Over recent years growing numbers of studies have extended our knowledge of two major types of target organ involvement in APS, namely those affecting the heart and the central nervous system (CNS). Heart valve lesions are the most common cardiac manifestations described in patients with APS. ${ }^{6-10}$ The introduction of cross sectional and Doppler echocardiography has revealed a high prevalence of valvar abnormalities_-such as thickening, stenosis, regurgitation, and vegetations-in patients with primary and secondary APS. Their frequency appears to be quite high, with up to $70 \%$ of APS patients having at least one valvar abnormality on echocardiography. ${ }^{411}$ Although many of these abnormalities are of little clinical consequence, vegetations of the mitral or aortic valves are present in approximately $4 \%$ of the patients, ${ }^{11}$ and $4-6 \%$ of all APS patients develop severe mitral or aortic regurgitation, half of whom require valve replacement surgery. ${ }^{8}$ Regarding the strong association of cardiac valve disease with APS, it has recently been suggested that cardiac valve thickening or vegetations should be included among the classification criteria for APS. ${ }^{13}$

CNS involvement is also one of the most prominent clinical manifestations of APS. Although the neurological involvement is thought to be thrombotic in origin, and cerebral ischaemia associated with antiphospholipid antibodies (aPL) is the most common arterial thrombotic manifestation, various other neuropsychiatric manifestations-including optic atrophy, chronic headache, dementia, cognitive dysfunction, psychosis, depression, transverse myelopathy, multiple sclerosis-like disease, chorea, and seizures-have also been associated with aPL. ${ }^{14} 15$

Despite the reported heterogeneity in clinical expression of APS, the interrelations between various manifestations of the disease have not yet been studied, and might have been overlooked owing to numerous possible combinations of clinical features. Awareness of such associations might give us a better understanding of the disease. Considering the high prevalences of cardiac and CNS manifestations in APS we sought to evaluate possible interassociations between these manifestations in a large group of APS patients.

\section{METHODS}

\section{Patients}

We recruited 284 APS patients from seven medical centres in Israel, Yugoslavia, and the Slovak Republic. All patients met the 1997 revised Sapporo criteria for the antiphospholipid syndrome. ${ }^{16}$ All underwent detailed medical interview and routine physical examination by a qualified internist or rheumatologist before inclusion in the study. Further data were obtained from the patients' medical files with respect to expression of the clinical manifestations of the disease, demographic variables, and laboratory results. The study was designed to evaluate possible associations between cardiac valve abnormalities and epilepsy or migraine. All patients underwent cross sectional and Doppler colour flow echocardiographic examination for determination of cardiac valve

Abbreviations: APL, antiphospholipid antibodies; APS, antiphospholipid syndrome; PAPS, primary antiphospholipid syndrome 
Table 1 Clinical manifestations in the study population

\begin{tabular}{|c|c|c|c|c|}
\hline Clinical manifestations & $\begin{array}{l}\text { Entire APS } \\
\text { population } \\
(\mathrm{n}=284)\end{array}$ & PAPS $(n=159)$ & $\begin{array}{l}\text { SLE with APS } \\
(\mathrm{n}=97)\end{array}$ & p Value \\
\hline Deep venous thrombosis & 57.4 & 52.2 & 59.8 & NS \\
\hline Arterial thrombosis & 48.6 & 45.3 & 56.7 & NS \\
\hline Recurrent pregnancy loss & 27.7 & 34.6 & 19.8 & $<0.03$ \\
\hline TIA/CVA & 35.2 & 35.8 & 39.2 & NS \\
\hline Myocardial infarction & 7.4 & 5.7 & 10.3 & NS \\
\hline Epilepsy & 9.9 & 6.9 & 15.5 & $<0.03$ \\
\hline Migraine & 18.0 & 20.8 & 14.4 & NS \\
\hline Cardiac valve vegetations & 6.0 & 4.4 & 9.3 & NS \\
\hline Cardiac valve thickening & 18.0 & 17.6 & 19.6 & NS \\
\hline Overall cardiac valve abnormalities & 21.1 & 18.2 & 27.8 & NS \\
\hline Livedo reticularis & 15.8 & 11.3 & 25.8 & $<0.005$ \\
\hline Thrombocytopenia & 28.5 & 22.6 & 40.2 & $<0.005$ \\
\hline Autoimmune haemolytic anaemia & 10.2 & 7.5 & 17.5 & $<0.02$ \\
\hline
\end{tabular}

abnormalities. Valve thickening was defined as focal areas of increased echogenicity and thickening of cardiac valve leaflets. For the mitral valve, a thickness of more than $5 \mathrm{~mm}$ was considered abnormal; for other valves abnormal thickness was determined according to standards of the specific medical centre. Valvar dysfunction was defined as the presence of stenosis or regurgitation of at least moderate severity. Non-infectious vegetations were defined as echocardiographically identified masses protruding from valve leaflets in the presence of sterile blood cultures and the absence of fever. Epilepsy was defined as at least two separate episodes of unprovoked seizures with an interval greater than 24 hours. Seizures could either be simple partial, complex partial, or generalised tonic-clonic, and had to be unrelated to metabolic abnormalities or to drug use or withdrawal, and without other provoking factors such as hypoxia or cerebral hypoperfusion. Migraine was defined by a neurologist at the specific medical centre as a recurrent severe unilateral or bilateral headache, lasting 4-72 hours, with or without aura, after excluding other relevant aetiologies such as tension headache or headache secondary to hypertension.

The cardiac-CNS associations were determined for the entire study population, and for subgroups of patients with primary APS (PAPS) or APS associated with systemic lupus erythematosus (SLE).

\section{Detection of anti-ק2GPI antibodies and anticardiolipin antibodies}

The levels of antiphospholipid antibodies were detected by enzyme linked immunosorbent assay (ELISA). Ninety six well ELISA plates (Nunc, Kamstrup, Roskilde, Denmark) were coated with cardiolipin (Sigma, St Louis, Missouri, USA), $50 \mu \mathrm{g} / \mathrm{ml}$ in ethanol, or $\beta 2$ glycoprotein I ( $\beta 2 \mathrm{GPI}), 10$ $\mu \mathrm{g} / \mathrm{ml}$ in phosphate buffered saline (PBS). Following blocking with $3 \%$ bovine serum albumin (BSA), sera at different dilutions were added and incubated for two hours at room temperature. Bound antibodies were detected using goat antihuman IgG or IgM conjugated to alkaline phosphatase (Sigma) and appropriate substrate. The colour reaction was read in a Titertrek ELISA reader (SLT Labistruments, Salzburg, Austria) at an optical density of $405 \mathrm{~nm}$. Extensive washing with PBS followed each step.

\section{Statistics}

Either the $\chi^{2}$ test or Fisher's exact test was applied as appropriate to analyse statistically significant differences between categorical variables. Two sided probability (p) values of less than 0.05 were considered significant.

\section{RESULTS}

We studied 284 APS patients, 238 of whom (83.8\%) were women and 46 (16.2\%) were men. The mean (SD) age of the

Table 2 Cardiac valve abnormalities in patients with antiphospholipid syndrome with or without CNS manifestations (epilepsy and migraine)

\begin{tabular}{|c|c|c|c|c|c|c|}
\hline & With epilepsy & $\begin{array}{l}\text { Without } \\
\text { epilepsy }\end{array}$ & p Value & $\begin{array}{l}\text { With } \\
\text { migraine }\end{array}$ & $\begin{array}{l}\text { Without } \\
\text { migraine }\end{array}$ & p Value \\
\hline \multicolumn{7}{|l|}{ (A) Whole study population } \\
\hline Cardiac valve vegetations & 17.9 & 4.7 & $<0.02$ & 11.8 & 4.7 & 0.09 \\
\hline Valvar thickening and/or dysfunction & 25.0 & 17.1 & NS & 33.3 & 14.6 & 0.002 \\
\hline Overall cardiac valve abnormalities & 32.1 & 19.9 & NS & 37.3 & 17.6 & 0.002 \\
\hline \multicolumn{7}{|l|}{ (B) PAPS } \\
\hline Cardiac valve vegetations & 18.1 & 3.5 & $<0.03$ & 12.1 & 2.4 & $<0.04$ \\
\hline Valvar thickening and/or dysfunction & 27.3 & 16.9 & NS & 33.3 & 13.5 & $<0.01$ \\
\hline Overall cardiac valve abnormalities & 27.3 & 17.6 & NS & 36.3 & 13.5 & 0.002 \\
\hline \multicolumn{7}{|l|}{ (C) APS associated with SLE } \\
\hline Cardiac valve vegetations & 13.3 & 8.5 & NS & 7.1 & 9.6 & NS \\
\hline Valvar thickening and/or dysfunction & 19.5 & 20.0 & NS & 21.4 & 19.2 & NS \\
\hline Overall cardiac valve abnormalities & 33.3 & 26.8 & NS & 28.6 & 27.7 & NS \\
\hline
\end{tabular}

Values are per cent.

(A): the whole study population ( $n=284)$; (B): patients with PAPS $(n=159)$; (C): patients with SLE and APS $(n=97)$

APS, antiphospholipid syndrome; PAPS, primary antiphospholipid syndrome; SLE, systemic lupus erythematosus. 
Table 3 Cardiac valve abnormalities in patients with antiphospholipid syndrome with and without cerebrovascular accident or transient ischaemic attack

\begin{tabular}{|c|c|c|c|}
\hline & $\begin{array}{l}\text { With } \\
\text { CVA/TIA }\end{array}$ & $\begin{array}{l}\text { Without } \\
\text { CVA/TIA }\end{array}$ & $\mathrm{p}$ Value \\
\hline \multicolumn{4}{|l|}{ (A) Whole study population } \\
\hline $\begin{array}{l}\text { Cardiac valve vegetations } \\
\text { Valvar thickening and/or }\end{array}$ & 10.0 & 3.8 & $<0.04$ \\
\hline dysfunction & 26.0 & 13.6 & $<0.01$ \\
\hline $\begin{array}{l}\text { Overall cardiac valve } \\
\text { abnormalities }\end{array}$ & 32.0 & 15.2 & $<0.001$ \\
\hline \multicolumn{4}{|l|}{ (B) PAPS } \\
\hline & 7.0 & 2.9 & NS \\
\hline $\begin{array}{l}\text { dysfunction } \\
\text { Overall cardiac valve }\end{array}$ & 26.3 & 12.7 & 0.03 \\
\hline abnormalities & 28.1 & 12.7 & $<0.02$ \\
\hline \multicolumn{4}{|l|}{ (C) APS associated with SLE } \\
\hline $\begin{array}{l}\text { Cardiac valve vegetations } \\
\text { Valvar thickening and/or }\end{array}$ & 13.1 & 6.8 & NS \\
\hline dysfunction & 23.7 & 16.9 & NS \\
\hline $\begin{array}{l}\text { Overall cardiac valve } \\
\text { abnormalities }\end{array}$ & 36.8 & 22.0 & NS \\
\hline \multicolumn{4}{|c|}{$\begin{array}{l}\text { Values are per cent. } \\
\text { (A): the whole study population }(n=284) \text {; (B): patients with PAPS } \\
(n=159) ;(C) \text { : patients with SLE and APS }(n=97) \text {. } \\
\text { APS, antiphospholipid syndrome; CVA, cerebrovascular accident; PAPS, } \\
\text { primary antiphospholipid syndrome; SLE, systemic lupus erythematosus; } \\
\text { TIA, transient ischaemic attack. }\end{array}$} \\
\hline
\end{tabular}

patients was 33.7 (11.9) years (range 16 to 71 ) and mean disease duration was 7.8 (5.2) years. Most patients had primary APS $(n=159,56 \%)$. APS was associated with SLE in 97 cases $(34.2 \%)$. The rates of anti-cardiolipin (IgG and/or $\operatorname{IgM}$ ) and anti- $\beta 2$ GPI (IgG and/or IgM) antibodies were $88.0 \%$ and $72.2 \%$, respectively. Lupus anticoagulants were detected in $77.1 \%$ of the study population. The prevalences of the most frequent clinical manifestations among the patients are presented in table 1 . The patients with APS associated with SLE had significantly higher rates of epilepsy, livedo reticularis, thrombocytopenia, and autoimmune haemolytic anaemia, and lower rates of fetal loss, than the patients with primary APS. There were 60 patients with valvar disease, of whom 17 had non-infectious vegetations and 51 had valve thickening or dysfunction. Some of the patients had several valvar abnormalities. Information about anticoagulant and anti-aggregate treatments was available for 235 patients. One hundred and nine patients received anticoagulant treatment; in 66 of these the international normalised ratio (INR) was targeted to $3-4$, and in 43 it was targeted to $2-3$. Fifty nine patients were treated with antiplatelet aggregants. There were no differences in the rates of anticoagulant or antiaggregate treatments in patients with valve disease with or without epilepsy or migraine.

The heart-CNS associations are detailed in table 2. When analysing the whole group of APS patients, significant associations where found between cardiac vegetations and epilepsy $(p<0.02)$, and between cardiac valve thickening or dysfunction-as well as overall valvar abnormalities (vegetations and/or valvar dysfunction) - and migraine $(p=0.002$ for both). Borderline association was found between valvar vegetations and migraine $(p=0.09)$. Subanalyses revealed striking differences between patients with primary APS (table 2B) and SLE patients with APS (table 2C). While patients with primary APS had significant associations between cardiac valve vegetations and epilepsy, and between any valvar abnormality (vegetations, dysfunction, or both) and migraine, patients with APS associated with SLE had no such associations. We also evaluated a possible connection between cardiac valve abnormalities and the occurrence of cerebrovascular accidents (CVA) or transient ischaemic attacks (TIA). As shown in table 3, significant associations were found between valvar disease and past episodes of CVA/ TIA (table 3A). These associations were confined to patients with primary APS and were not found in patients with APS associated with SLE (table 3B and 3C, respectively).

\section{DISCUSSION}

In this study we have identified significant associations between cardiac valve lesions and CNS manifestations in a large group of APS patients. The presence of valvar vegetations was found to be associated with epilepsy and migraine, while valvar thickening or dysfunction, as well as overall valvar abnormalities, were significantly associated with the presence of migraine. Furthermore, cardiac valve vegetations or dysfunction, or both, were strongly associated with a high rate of CVA or TIA. The rate of cardiac valve abnormalities in our study population was $21.1 \%$. It was somewhat more common in SLE patients $(27.8 \%)$ than in primary APS (18.2\%, NS). The prevalence of valvar pathology in our study is in the range reported in previous studies, ${ }^{7}$ ranging usually between $32 \%$ and $38 \%$ of APS patients, ${ }^{8} 9111718$ although lower and higher prevalences have also been reported. ${ }^{9}$ Recently, Zavaleta et al ${ }^{12}$ reported a $70.8 \%$ rate of valvar lesions in APS patients, detected by transoesophageal echocardiography. New valvar lesions or progression of existing lesions were detected in nine of 12 patients during a five year follow up. ${ }^{12}$ Epilepsy was diagnosed in $9.9 \%$ of the patients in our study, and migraine in $18.0 \%$-in line with previously reported rates. ${ }^{19}{ }^{20}$ In the current study we did not have information on the sequence of events. Thus, further prospective studies are needed to evaluate whether cardiac valvar disease is a risk factor for CNS manifestations, or vice versa.

A possible link between heart pathology and various CNS involvements in APS has not yet been investigated. The only cardiac-CNS association in APS to be reported thus fur has been on valve vegetations as a possible risk factor for cerebral emboli. ${ }^{21-23}$ The results of our study support this observation. To the best of our knowledge a relation between valvar vegetations or dysfunction and other CNS manifestations has not yet been reported.

The pathogenesis of cardiac valve and cerebral abnormalities in APS is not entirely clear. It has been postulated that aPL cause valvar or endothelial injury directly, independently of the severity of the disease, with aPL deposition on the valves resembling immune complex deposition in the dermoepidermal junction or in the kidney basement membrane in patients with SLE. ${ }^{10} 24$ Antibody deposition and complement components initiating valve damage have been described, ${ }^{6}$ along with increased endothelial cell expression of $\alpha 3 \beta 1$ integrin. ${ }^{25}$ On the other hand, some histological studies suggest that fibrin deposits are the major finding, not inflammation. ${ }^{26}$ It had also been suggested that anticoagulation may improve cardiac valve lesions, though other studies did not show benefit of anticoagulant or antiaggregate therapy..$^{13}$ In our study we did not find a relation between anticoagulant/antiaggregate treatment and the rates of valvar pathology.

As to CNS involvement, the specific role of aPL remains one of the least understood aspects of this syndrome. The link between aPL and epilepsy has been supported by several studies, ${ }^{27}{ }^{28}$ but there is as yet no direct evidence that aPL per se are pathogenic or are direct mediators in the development of thrombotic or other neurological complications in human patients. Laboratory findings which indicate that aPL directly bind to brain tissue, inhibit astrocytes proliferation in vitro, 
or depolarise brain nerve terminals may provide a basis for understanding the pathogenesis of epilepsy and other neurological complications in APS. ${ }^{29}{ }^{30}$ The pathophysiology of migraine is complex and includes genetic, metabolic, vascular, and emotional components. Cuadrado et $a l^{31}$ reported on five APS patients with intractable headaches treated with a seven day course of anticoagulation. All five patients had a marked improvement of the headaches following the treatment. It was speculated that low dose anticoagulant therapy might be tried in APS patients with migraine. ${ }^{31}$ Our study was not designed to evaluate the role of treatment on CNS or cardiac manifestations in APS, but we observed similar rates of migraine in patients treated or untreated with either anticoagulants or antiaggregate agents. The linkage between cardiac valve and CNS pathologies found in our study suggests a common pathogenic mechanism mediated, for example, by localised aPL associated tissue damage. Further studies are required to evaluate these mechanisms.

Of special interest are the opposite cardiac-CNS relations found between patients with primary APS and those with APS associated with SLE. Whereas primary APS patients showed a significant association between cardiac valve pathology and CNS manifestations, such associations were not found in patients with APS associated with SLE. Possible disparities in clinical presentation between patients with primary and secondary APS have not yet been thoroughly investigated. In a retrospective study, Asherson et al reported on a lower female to male sex ratio and lower rates of livedo reticularis and chorea in primary APS than in patients with SLE. ${ }^{32}$ Vianna et al found similar clinical and laboratory profiles in patients with primary APS or APS associated with SLE, with the exception that autoimmune haemolytic anaemia, endocardial valve disease, neutropenia, and low C4 levels all occurred more often in SLE patients. ${ }^{11}$ The different valvar-CNS connections found in our study may reflect distinct pathogenic mechanisms underlying cardiac or cerebral involvements in APS patients, depending on their background autoimmune condition.

In conclusion, we report on a close association between cardiac valve pathology and several CNS manifestations, such as epilepsy, migraine, and CVA/TIA, in patients with APS. Our study also suggests that there are distinct biological behaviours in primary APS and in APS associated with SLE. From our results, the presence of cardiac valve pathology may be a risk factor for several types of CNS involvement in primary APS.

\footnotetext{
Authors' affiliations

I Krause, M Blank, Y Shoenfeld, Research Centre for Autoimmune

Diseases, Sheba Medical Centre, Tel-Hashomer and Sackler Faculty of Medicine, Tel-Aviv University, Israel

S Lev, A Fraser, Department of Medicine "E", Rabin Medical Centre, Sackler Faculty of Medicine, Tel-Aviv University, Israel

M Lorber, Institute of Allergy, Clinical Immunology and AIDS, Rambam Medical Centre and Rappaport Faculty of Medicine, Technion, Israel L Stojanovich, Institute of Rheumatology, University Hospital, Belgrade, Yugoslavia

J Rovensky, National Institute of Rheumatic Diseases, Bratislava, Slovak Republic

J Chapman, Department of Neurology, Sheba Medical Centre

\section{REFERENCES}

1 Alarcon Segovia D, Deleze M, Oria CV, Sanchez-Guerrero J, GomezPacheco L, Cabiedes J, et al. Antiphospholipid antibodies and the antiphospholipid syndrome in systemic lupus erythematosus. A prospective analysis of 500 consecutive patients. Medicine (Baltimore) 1989;68:353-65. 2 Hughes GR, Harris NN, Gharavi AE. The anticardiolipin syndrome. $J$ Rheumatol 1986;13:486-9.
}

3 Alarcon Segovia D. The antiphospholipid story. J Rheumatol 2003;30:1893-6.

4 Levine JS, Branch DW, Rauch J. The antiphospholipid syndrome. N Engl J Med 2002;346:752-63.

5 Shoenfeld Y. Systemic antiphospholipid syndrome. Lupus 2003;12:497-8.

6 Lev S, Shoenfeld Y. Cardiac valvulopathy in the antiphospholipid syndrome. Clin Rev Allergy Immunol 2002;23:341-8.

7 Cervera R. Recent advances in antiphospholipid antibody-related valvulopathies. J Autoimmun 2000;15:123-5.

8 Nesher G, llany J, Rosenmann D, Abraham AS. Valvular dysfunction in antiphospholipid syndrome: prevalence, clinical features, and treatment. Semin Arthritis Rheum 1997;27:27-35.

9 Hojnik M, George J, Ziporen L, Shoenfeld Y. Heart valve involvement (LibmanSacks endocarditis) in the antiphospholipid syndrome. Circulation 1996;93:1579-87.

10 Ziporen L, Goldberg I, Arad M, Hojnik M, Ordi-Ros J, Afek A, et al. LibmanSacks endocarditis in the antiphospholipid syndrome: immunopathologic findings in deformed heart valves. Lupus 1996;5:196-205.

11 Vianna JL, Khamashta MA, Ordi Ros J, Font J, Cervera R, Lopez-Soto A, et al. Comparison of the primary and secondary antiphospholipid syndrome: a European multicenter study of 114 patients. Am J Med 1994;96:3-9.

12 Zavaleta NE, Montes RM, Soto ME, Vanzzini NA, Amigo MC. Primary antiphospholipid syndrome: a 5-year transesophageal echocardiographic followup study. J Rheumatol 2004;31:2402-7.

13 Petri MA. Classification criteria for antiphospholipid syndrome: the case for cardiac valvular disease. J Rheumatol 2004;31:2329-30.

14 Sanna G, Bertolaccini ML, Cuadrado MJ, Khamashta MA, Hughes GR. Central nervous system involvement in the antiphospholipid (Hughes) syndrome. Rheumatology (Oxford) 2003;42:200-13.

15 Hughes GR. Migraine, memory loss, and "multiple sclerosis". Neurological features of the antiphospholipid (Hughes') syndrome. Postgrad Med J 2003;79:81-3.

16 Wilson WA, Gharavi AE, Koike T, Lockshin MD, Branch DW, Piette JC, et al. International consensus statement on preliminary classification criteria for definite antiphospholipid syndrome: report of an international workshop. Arthritis Rheum 1999;42:1309-11.

17 Galve E, Ordi J, Barquinero J, Evangelista A, Vilardell M, Soler Soler J. Valvular heart disease in the primary antiphospholipid syndrome. Ann Intern Med 1992;1 16:293-8.

18 Cervera R, Khamashta MA, Font J, Reyes PA, Vianna JL, Lopez-Soto A, et al. High prevalence of significant heart valve lesions in patients with the 'primary' antiphospholipid syndrome. Lupus 1991;1:43-7.

19 Cervera R, Piette JC, Font J, Khamashta MA, Shoenfeld Y, Camps MT, et al. Antiphospholipid syndrome: clinical and immunologic manifestations and patterns of disease expression in a cohort of 1,000 patients. Arthritis Rheum 2002;46:1019-27.

20 Shoenfeld Y, Lev S, Blatt I, Blank M, Font J, von Landenberg P, et al. Features associated with epilepsy in the antiphospholipid syndrome. $J$ 'Rheumatol 2004;31:1344-8.

21 Pope JM, Canny CL, Bell DA. Cerebral ischemic events associated with endocarditis, retinal vascular disease, and lupus anticoagulant. Am J Med 1991;90:299-309.

22 Barbut D, Borer JS, Gharavi A, Wallerson D, Devereux RB, Supino P, et al. Prevalence of anticardiolipin antibody in isolated mitral or aortic regurgitation, or both, and possible relation to cerebral ischemic events. Am J Cardiol 1992;70:901-5.

23 Pamuk ON, Cakir N, Soy M, Aktoz M, Celik Y, Akdemir O. Mitral valve vegetation and cerebral emboli in a primary antiphospholipid syndrome patient who had hepatitis $C$ virus infection: report of a case and review of the literature. Clin Rheumatol 2003;22:136-9.

24 Amital H, Langevitz P, Levy Y, Afek A, Goldberg I, Pras M, et al. Valvular deposition of antiphospholipid antibodies in the antiphospholipid syndrome: a clue to the origin of the disease. Clin Exp Rheumatol 1999;17:99-102.

25 Afek A, Shoenfeld Y, Manor R, Goldberg I, Ziporen L, George J, et al. Increased endothelial cell expression of alpha3betal integrin in cardiac valvulopathy in the primary (Hughes) and secondary antiphospholipid syndrome. Lupus 1999;8:502-7.

26 Garcia Torres R, Amigo MC, de la Rosa A, Moron A, Reyes PA. Valvular heart disease in primary antiphospholipid syndrome (PAPS): clinical and morphological findings. Lupus 1996;5:56-61.

27 Peltola JT, Haapala A, Isojarvi J, Auvinen A, Palmio J, Latvala K, et al. Antiphospholipid and antinuclear antibodies in patients with epilepsy or newonset seizure disorders. Am J Med 2000;109:712-17.

28 Chapman J, Rand JH, Brey RL, Levine SR, Blatt I, Khamashta MA, et al. Nonstroke neurological syndromes associated with antiphospholipid antibodies: evaluation of clinical and experimental studies. Lupus 2003;12:514-17.

29 Sun KH, Liu WT, Tsai CY, Liao TS, Lin WM, Yu CL. Inhibition of astrocyte proliferation and binding to brain tissue of anticardiolipin antibodies purified from lupus serum. Ann Rheum Dis 1992;51:707-12.

30 Chapman J, Cohen Armon M, Shoenfeld Y, Korczyn AD. Antiphospholipid antibodies permeabilize and depolarize brain synaptoneurosomes. Lupus 1999;8:127-33.

31 Cuadrado MJ, Khamashta MA, Hughes GR. Migraine and stroke in young women. QJM 2000;93:317-18.

32 Asherson RA, Khamashta MA, Ordi Ros J, Derksen RH, Machin SJ, Barquinero J, et al. The "primary" antiphospholipid syndrome: major clinical and serological features. Medicine (Baltimore) 1989;68:366-74. 